\title{
Galilei invariance of the Helmholtz morphism
}

\author{
Radka Malíková
}




\title{
GALILEI INVARIANCE OF THE HELMHOLTZ MORPHISM
}

\section{RADKA MALÍKOVÁ}

\begin{abstract}
We study the Helmholtz morphism in terms of the variational sequences. We find conditions for the Helmholtz form to be invariant with respect to the Galilei group.
\end{abstract}

2000 Mathematics Subject Classification: 34C40, 70H33

Keywords: Variational sequence, Helmholtz morphism, symmetry, Galilei group

\section{INTRODUCTION}

In this paper, we shall use the framework of the theory of variational sequences on fibred manifolds introduced by Krupka [2,3]. The variational sequence is a quotient sequence of the de Rham sequence such that one of the morphisms is the EulerLagrange morphism $\varepsilon_{1}: \lambda \rightarrow E_{\lambda}$, assigning to a Lagrangian, i.e. one-form $\lambda=$ $L \mathrm{~d} t$, its Euler-Lagrange form, i. e. two-form $E_{\lambda}=E_{\sigma}(L) \mathrm{d} q^{\sigma} \wedge \mathrm{d} t$, where $E_{\sigma}(L)$ are the Euler-Lagrange expressions

$$
E_{\sigma}=\frac{\partial L}{\partial q^{\sigma}}-\frac{\mathrm{d}}{\mathrm{d} t} \frac{\partial L}{\partial \dot{q}^{\sigma}} .
$$

The next morphism $\varepsilon_{2}: E \rightarrow H_{E}$, called the Helmholtz morphism, assigns to a twoform $E=E_{\sigma} \mathrm{d} q^{\sigma} \wedge \mathrm{d} t$ a three-form $H_{E}$

$$
\begin{aligned}
H_{E}= & \frac{1}{2}\left(\frac{\partial E_{\sigma}}{\partial q^{v}}-\frac{\partial E_{v}}{\partial q^{\sigma}}-\frac{1}{2} \frac{\mathrm{d}}{\mathrm{d} t}\left(\frac{\partial E_{\sigma}}{\partial \dot{q}^{v}}-\frac{\partial E_{v}}{\partial \dot{q}^{\sigma}}\right)\right) \omega^{v} \wedge \omega^{\sigma} \wedge \mathrm{d} t \\
& +\frac{1}{2}\left(\frac{\partial E_{\sigma}}{\partial \dot{q}^{v}}+\frac{\partial E_{v}}{\partial \dot{q}^{\sigma}}-\frac{\mathrm{d}}{\mathrm{d} t}\left(\frac{\partial E_{\sigma}}{\partial \ddot{q}^{v}}+\frac{\partial E_{v}}{\partial \ddot{q}^{\sigma}}\right)\right) \dot{\omega}^{v} \wedge \omega^{\sigma} \wedge \mathrm{d} t \\
& +\frac{1}{2}\left(\frac{\partial E_{\sigma}}{\partial \ddot{q}^{v}}-\frac{\partial E_{v}}{\partial \ddot{q}^{\sigma}}\right) \ddot{\omega}^{v} \wedge \omega^{\sigma} \wedge \mathrm{d} t .
\end{aligned}
$$

called Helmholtz form.

While the Euler-Lagrange morphism $\mathscr{E}_{1}$ is well-understood, much less is known about the Helmholtz morphism. In the present paper, we study the Galilei invariance of the Helmholtz morphism.

In Section 2, we recall the basic structures and notations and briefly introduce the variational sequence, according to [2,3,5], and we recall known properties of the 
Helmholtz morphism [6,7]. In Section 3, we recall the basic concepts of the theory of symmetries of differential forms, the Noether theorem, and the Galilei group of transformations. We present a theorem on the structure of Helmholtz forms invariant with respect to the Galilei group.

\section{THE HeLMHOLTZ MORPHISM}

Let $\pi: Y \rightarrow X$ be a smooth fibred manifold, $\operatorname{dim} X=1, \operatorname{dim} Y=m+1$, and $\pi_{r}: J^{r} Y \rightarrow X, r \geq 1$, its jet prolongations. Denote by $\pi_{r, s}: J^{r} Y \rightarrow J^{s} Y$, $r>s \geq 0$, canonical jet projections. A mapping $\gamma: W \rightarrow Y$, where $W$ is an open subset of $X$, is called a section of the manifold $\pi: Y \rightarrow X$ if $\gamma \circ \pi=\mathrm{id}_{W}$.

Fibred coordinates on $Y$ are denoted by $\left(t, q^{\sigma}\right), 1 \leq \sigma \leq m$, associated coordinates on $J^{r} Y$ are denoted by $\left(t, q_{i}^{\sigma}\right), 1 \leq \sigma \leq m, 0 \leq i \leq r$. We usually use the notation $q_{0}^{\sigma}=q^{\sigma}, q_{1}^{\sigma}=\dot{q}^{\sigma}, q_{2}^{\sigma}=\ddot{q}^{\sigma}, q_{3}^{\sigma}=\dddot{q}^{\sigma}$.

A vector field $\xi$ on $J^{r} Y$ is called $\pi_{r}$-vertical if $T \pi_{r} \cdot \xi=0$, and $\pi_{r}$-projectable if there exists a vector field $\xi_{0}$ on $X$ such that $T \pi_{r} \cdot \xi=\xi_{0} \circ \pi_{r}$.

A differential $q$-form $(q>1) \eta$ on $J^{r} Y$ is called contact if $J^{r} \gamma^{*} \eta=0$ for every section $\gamma$ of $\pi$, horizontal or 0 -contact if $i_{\xi} \eta=0$ for every vertical vector field $\xi$ on $J^{r} Y$, and $k$-contact, $1 \leq k \leq q$, if for every vertical vector field $\xi, i_{\xi} \eta$ is $(k-1)$ contact. If lifted to $J^{r+1} Y$, every $q$-form $\eta$ on $J^{r} Y$ can be canonically decomposed into a sum of $k$-contact components, $\eta_{k}$, where $k=0,1, \ldots, q$. We write $\eta_{k}=p_{k} \eta$, and $p_{0}=h$, then,

$$
\pi_{r+1, r}^{*} \eta=h \eta+p_{1} \eta+\cdots+p_{q} \eta
$$

A contact $q$-form is called strongly contact if $\pi_{r+1, r}^{*} \eta=p_{q} \eta$.

A general framework for our exposition is the variational sequence [2,3].

Let $\Omega_{0, c}^{r}=\{0\}$, and let $\Omega_{p, c}^{r}$ be the sheaf of contact $p$-forms, if $p \leq n$, or the sheaf of strongly contact $p$-forms, if $p>n$, on $J^{r} Y$. Set

$$
\Theta_{p}^{r}=\Omega_{p, c}^{r}+\mathrm{d} \Omega_{p-1, c}^{r},
$$

where $\mathrm{d} \Omega_{p-1, c}^{r}$ is the image sheaf of $\Omega_{p-1, c}^{r}$ by the exterior derivative d. We get an exact sequence of soft sheaves

$$
0 \longrightarrow \Theta_{1}^{r} \longrightarrow \Theta_{2}^{r} \longrightarrow \Theta_{3}^{r} \longrightarrow \cdots,
$$

where the morphisms are the exterior derivative, i. e., a subsequence of the De Rham seguence

$$
0 \longrightarrow \mathbb{R} \longrightarrow \Omega_{0}^{r} \longrightarrow \Omega_{1}^{r} \longrightarrow \Omega_{2}^{r} \longrightarrow \Omega_{3}^{r} \longrightarrow \cdots
$$

The quotient sequence

$$
0 \longrightarrow \mathbb{R} \longrightarrow \Omega_{0}^{r} \longrightarrow \Omega_{1}^{r} / \Theta_{1}^{r} \longrightarrow \Omega_{2}^{r} / \Theta_{2}^{r} \longrightarrow \Omega_{3}^{r} / \Theta_{3}^{r} \longrightarrow \cdots
$$

is also exact. It is called the variational sequence of order $r$ on $\pi$. The variational sequence is an acyclic resolution of the constant sheaf $\mathbb{R}$ over $Y$. We denote by

$$
\varepsilon_{p}: \Omega_{p}^{r} / \Theta_{p}^{r} \rightarrow \Omega_{p+1}^{r} / \Theta_{p+1}^{r}
$$


the quotient mapping. The class of a form $\rho \in \Omega_{p}^{r}$ is denoted by $[\rho]$. Hence, $\varepsilon_{p}([\rho])=[\mathrm{d} \rho]$.

The quotient mapping

$$
\varepsilon_{1}: \Omega_{1}^{r} / \Theta_{1}^{r} \rightarrow \Omega_{2}^{r} / \Theta_{2}^{r}
$$

then identifies with the Euler-Lagrange mapping. The quotient mapping

$$
\varepsilon_{2}: \Omega_{2}^{r} / \Theta_{2}^{r} \rightarrow \Omega_{3}^{r} / \Theta_{3}^{r}
$$

is called the Helmholtz mapping. The image of a class $[\rho] \in \Omega_{2}^{r} / \Theta_{2}^{r}$, i. e., the class $[\mathrm{d} \rho] \in \Omega_{3}^{r} / \Theta_{3}^{r}$ is called Helmholtz class.

Due to the exactness of the variational sequence, if $[\alpha] \in \Omega_{2}^{r} / \Theta_{2}^{r}$ is such that

$$
\varepsilon_{2}([\alpha])=[d \alpha]=0,
$$

there exists $[\rho] \in \Omega_{1}^{r} / \Theta_{1}^{r}$ such that $[\alpha]=[d \rho]=\mathcal{E}_{1}([\rho])$, i. e. $[\alpha]$ is the image by the Euler-Lagrange mapping of a class $[\rho]$. In other words, the class $[\alpha]$ is locally variational - it comes from a class $[\rho]$ that has the meaning of a local Lagrangian. If, moreover, $H^{2} Y=\{0\}$, a global Lagrangian exists. Condition (2.2) for "local variationality" then provides Helmholtz conditions (of order $r$ ).

Classes in the variational sequence can be represented by differential forms $[1,4]$. We shall use the representation by so-called source forms, $(q-1)$-contact $q$-forms belonging to the ideal generated by contact forms

$$
\omega^{\sigma}=\mathrm{d} q^{\sigma}-\dot{q}^{\sigma} \mathrm{d} t, \quad \dot{\omega}^{\sigma}=\mathrm{d} \dot{q}^{\sigma}-\ddot{q}^{\sigma} \mathrm{d} t, \quad \cdots, \quad \omega_{r-1}^{\sigma}=\mathrm{d} q_{r-1}^{\sigma}-\dot{q}_{r}^{\sigma} \mathrm{d} t
$$

where $1 \leq i \leq m$.

Source forms for classes $[\rho] \in \Omega_{1}^{r} / \Theta_{1}^{r}$ are horizontal forms $\lambda=L \mathrm{~d} t$, called Lagrangians. Source forms for classes $[\alpha] \in \Omega_{2}^{r} / \Theta_{2}^{r}$ are two-forms $E=E_{i} \omega^{i} \wedge \mathrm{d} t$, called dynamical forms (corresponding to differential equations). Note that, in this representation, if $[\rho]$ is represented by $\lambda$, then $[\mathrm{d} \rho]=\mathcal{E}_{1}([\rho])$ is represented by the dynamical form $E_{\lambda}$, the Euler-Lagrange form of $\lambda$. If $[\alpha] \in \Omega_{2}^{r} / \Theta_{2}^{r}$ is represented by a dynamical form $E,[\mathrm{~d} \alpha]=\mathcal{E}_{2}([\alpha])$ is represented by a source three-form $H_{E}$, the Helmholtz-form of $E$. As shown in [3], $H_{E}$ is then given by (1.1).

Source forms representing elements in $\Omega_{3}^{r} / \Theta_{3}^{r}$ are called Helmholtz-like forms. We shall be interested in Helmholtz-like forms of order 3 (in particular, they correspond to second order ordinary differential equations). In coordinates,

$$
H=H_{\sigma \nu}^{0} \omega^{v} \wedge \omega^{\sigma} \wedge \mathrm{d} t+H_{\sigma \nu}^{1} \dot{\omega}^{\nu} \wedge \omega^{\sigma} \wedge \mathrm{d} t+H_{\sigma v}^{2} \ddot{\omega}^{\nu} \wedge \omega^{\sigma} \wedge \mathrm{d} t
$$

where $H_{\sigma \nu}^{0}=-H_{\nu \sigma}^{0}, H_{\sigma \nu}^{1}=H_{\nu \sigma}^{1}, H_{\sigma \nu}^{2}=-H_{\nu \sigma}^{2}$.

In $[6,7]$ was studied and solved the question when a three-form corresponds to a system of differential equations (such form is called locally Helmholtz). This problem is closely related to the question of existence of a closed counterpart of a three-form. This problem is solved for second-order Helmholtz-like forms in [6] and for thirdorder Helmholtz-like forms in [7]. 


\section{GALilei INVARIANCE OF THE HelmHOLTZ FORM}

Let $\xi$ be a projectable vector field on $Y, \eta$ a differential form on $J^{r} Y$. $\xi$ is called point symmetry of $\eta$, if

$$
\partial_{J}{ }^{r} \eta=0
$$

where $\partial$ denotes the Lie derivative. If $\eta$ is a Helmholtz-like form of order three (2.4) the symmetry conditions (3.1) reads as follows:

$$
\begin{aligned}
& 0=H_{\sigma \rho}^{0} \frac{\partial \xi^{\rho}}{\partial q^{v}}+H_{\rho \nu}^{0} \frac{\partial \xi^{\rho}}{\partial q^{\sigma}}+H_{\sigma \nu}^{0} \frac{\partial \xi^{0}}{\partial t}+H_{\sigma \rho}^{1} \frac{\partial}{\partial q^{v}}\left(\frac{\mathrm{d} \xi^{\rho}}{\mathrm{d} t}-\dot{q}^{\rho} \frac{\mathrm{d} \xi^{0}}{\mathrm{~d} t}\right) \\
& +H_{\sigma \rho}^{2} \frac{\partial}{\partial q^{\nu}}\left(\frac{\mathrm{d}^{2} \xi^{\rho}}{\mathrm{d} t^{2}}-\frac{\mathrm{d}^{2} \xi^{0}}{\mathrm{~d} t^{2}} \dot{q}^{\rho}-2 \frac{\mathrm{d} \xi^{0}}{\mathrm{~d} t} \ddot{q}^{\rho}\right) \\
& +\frac{\partial H_{\sigma v}^{0}}{\partial t} \xi^{0}+\frac{\partial H_{\sigma v}^{0}}{\partial q^{\rho}} \xi^{\rho}+\frac{\partial H_{\sigma v}^{0}}{\partial \dot{q}^{\rho}}\left(\frac{\mathrm{d} \xi^{\rho}}{\mathrm{d} t}-\dot{q}^{\rho} \frac{\mathrm{d} \xi^{0}}{\mathrm{~d} t}\right) \\
& +\frac{\partial H_{\sigma v}^{0}}{\partial \ddot{q}^{\rho}}\left(\frac{\mathrm{d}^{2} \xi^{\rho}}{\mathrm{d} t^{2}}-\frac{\mathrm{d}^{2} \xi^{0}}{\mathrm{~d} t^{2}} \dot{q}^{\rho}-2 \frac{\mathrm{d} \xi^{0}}{\mathrm{~d} t} \ddot{q}^{\rho}\right) \\
& +\frac{\partial H_{\sigma \nu}^{0}}{\partial \dddot{q}^{\rho}}\left(\frac{\mathrm{d}^{3} \xi^{\rho}}{\mathrm{d} t^{3}}-\frac{\mathrm{d}^{3} \xi^{0}}{\mathrm{~d} t^{3}} \dot{q}^{\rho}-3 \frac{\mathrm{d}^{2} \xi^{0}}{\mathrm{~d} t^{2}} \ddot{q}^{\rho}-3 \frac{\mathrm{d} \xi^{0}}{\mathrm{~d} t} \dddot{q}^{\rho}\right) \\
& 0=H_{\sigma \rho}^{0} \frac{\partial \xi^{\rho}}{\partial \dot{q}^{v}}-H_{\rho \sigma}^{0} \frac{\partial \xi^{\rho}}{\partial \dot{q}^{v}} \\
& +H_{\sigma \nu}^{1} \frac{\partial \xi^{0}}{\partial t}+H_{\rho v}^{1} \frac{\partial \xi^{\rho}}{\partial q^{\sigma}}+H_{\sigma \rho}^{1} \frac{\partial}{\partial \dot{q}^{\nu}}\left(\frac{\mathrm{d} \xi^{\rho}}{\mathrm{d} t}-\dot{q}^{\rho} \frac{\mathrm{d} \xi^{0}}{\mathrm{~d} t}\right) \\
& +H_{\sigma \rho}^{2} \frac{\partial}{\partial \dot{q}^{\nu}}\left(\frac{\mathrm{d}^{2} \xi^{\rho}}{\mathrm{d} t^{2}}-\frac{\mathrm{d}^{2} \xi^{0}}{\mathrm{~d} t^{2}} \dot{q}^{\rho}-2 \frac{\mathrm{d} \xi^{0}}{\mathrm{~d} t} \ddot{q}^{\rho}\right) \\
& +\frac{\partial H_{\sigma v}^{1}}{\partial t} \xi^{0}+\frac{\partial H_{\sigma v}^{1}}{\partial q^{\rho}} \xi^{\rho}+\frac{\partial H_{\sigma v}^{1}}{\partial \dot{q}^{\rho}}\left(\frac{\mathrm{d} \xi^{\rho}}{\mathrm{d} t}-\dot{q}^{\rho} \frac{\mathrm{d} \xi^{0}}{\mathrm{~d} t}\right) \\
& +\frac{\partial H_{\sigma v}^{1}}{\partial \ddot{q}^{\rho}}\left(\frac{\mathrm{d}^{2} \xi^{\rho}}{\mathrm{d} t^{2}}-\frac{\mathrm{d}^{2} \xi^{0}}{\mathrm{~d} t^{2}} \dot{q}^{\rho}-2 \frac{\mathrm{d} \xi^{0}}{\mathrm{~d} t} \ddot{q}^{\rho}\right) \\
& +\frac{\partial H_{\sigma \nu}^{1}}{\partial \dddot{q}^{\rho}}\left(\frac{\mathrm{d}^{3} \xi^{\rho}}{\mathrm{d} t^{3}}-\frac{\mathrm{d}^{3} \xi^{0}}{\mathrm{~d} t^{3}} \dot{q}^{\rho}-3 \frac{\mathrm{d}^{2} \xi^{0}}{\mathrm{~d} t^{2}} \ddot{q}^{\rho}-3 \frac{\mathrm{d} \xi^{0}}{\mathrm{~d} t} \dddot{q}^{\rho}\right) \\
& 0=H_{\sigma \rho}^{0} \frac{\partial \xi^{\rho}}{\partial \ddot{q}^{v}}-H_{\rho \sigma}^{0} \frac{\partial \xi^{\rho}}{\partial \ddot{q}^{v}}+H_{\sigma \rho}^{1} \frac{\partial}{\partial \ddot{q}^{v}}\left(\frac{\mathrm{d} \xi^{\rho}}{\mathrm{d} t}-\dot{q}^{\rho} \frac{\mathrm{d} \xi^{0}}{\mathrm{~d} t}\right) \\
& +H_{\sigma v}^{2} \frac{\partial \xi^{0}}{\partial t}+H_{\rho v}^{2} \frac{\partial \xi^{\rho}}{\partial q^{\sigma}}+H_{\sigma \rho}^{2} \frac{\partial}{\partial \ddot{q}^{v}}\left(\frac{\mathrm{d}^{2} \xi^{\rho}}{\mathrm{d} t^{2}}-\frac{\mathrm{d}^{2} \xi^{0}}{\mathrm{~d} t^{2}} \dot{q}^{\rho}-2 \frac{\mathrm{d} \xi^{0}}{\mathrm{~d} t} \ddot{q}^{\rho}\right) \\
& +\frac{\partial H_{\sigma v}^{2}}{\partial t} \xi^{0}+\frac{\partial H_{\sigma v}^{2}}{\partial q^{\rho}} \xi^{\rho}+\frac{\partial H_{\sigma v}^{2}}{\partial \dot{q}^{\rho}}\left(\frac{\mathrm{d} \xi^{\rho}}{\mathrm{d} t}-\dot{q}^{\rho} \frac{\mathrm{d} \xi^{0}}{\mathrm{~d} t}\right)
\end{aligned}
$$




$$
\begin{aligned}
& +\frac{\partial H_{\sigma v}^{2}}{\partial \ddot{q}^{\rho}}\left(\frac{\mathrm{d}^{2} \xi^{\rho}}{\mathrm{d} t^{2}}-\frac{\mathrm{d}^{2} \xi^{0}}{\mathrm{~d} t^{2}} \dot{q}^{\rho}-2 \frac{\mathrm{d} \xi^{0}}{\mathrm{~d} t} \ddot{q}^{\rho}\right) \\
& +\frac{\partial H_{\sigma v}^{2}}{\partial \ddot{q}^{\rho}}\left(\frac{\mathrm{d}^{3} \xi^{\rho}}{\mathrm{d} t^{3}}-\frac{\mathrm{d}^{3} \xi^{0}}{\mathrm{~d} t^{3}} \dot{q}^{\rho}-3 \frac{\mathrm{d}^{2} \xi^{0}}{\mathrm{~d} t^{2}} \ddot{q}^{\rho}-3 \frac{\mathrm{d} \xi^{0}}{\mathrm{~d} t} \dddot{q}^{\rho}\right)
\end{aligned}
$$

The Galilei group on $\mathbb{R}^{4}$ is the 10-parametric transformation group, generated by the vector fields

$$
\frac{\partial}{\partial t}, \quad \frac{\partial}{\partial q^{\sigma}}, \quad-\varepsilon_{\sigma \nu \rho} q^{v} \frac{\partial}{\partial q^{\rho}}, \quad t \frac{\partial}{\partial q^{\sigma}}, \quad \sigma=1,2,3
$$

for the time translation, the space translations, the space rotations, and the Galilei transformations, respectively.

Theorem 1. A Helmholtz-like form $H$ (2.4) is invariant under the Galilei group of transformations if and only if the component $H_{\sigma \nu}^{0}$ of $H$ takes the form:

$$
H_{\sigma \nu}^{0}=\varepsilon_{\sigma \nu \rho} f(\eta) \ddot{q}^{\rho}+\varepsilon_{\sigma \nu \rho} g(\bar{\eta}) \ddot{q}^{\rho}
$$

where $\eta=\sum_{\kappa=1}^{3}\left(\ddot{q}^{\kappa}\right)^{2}, \bar{\eta}=\sum_{\kappa=1}^{3}\left(\dddot{q}^{\kappa}\right)^{2}$, and components $H_{\sigma v}^{1}, H_{\sigma \nu}^{2}$ of $H$ vanish.

Proof. Substituing the generators of the Galilei group (3.5) into (3.2)-(3.4) we obtain

$$
\begin{array}{llll}
\frac{\partial H_{\sigma v}^{0}}{\partial t}=0 & \frac{\partial H_{\sigma v}^{0}}{\partial q^{\rho}}=0 & \frac{\partial H_{\sigma v}^{0}}{\partial \dot{q}^{\rho}}=0 \\
\frac{\partial H_{\sigma v}^{1}}{\partial t}=0 & \frac{\partial H_{\sigma v}^{1}}{\partial q^{\rho}}=0 & \frac{\partial H_{\sigma v}^{1}}{\partial \dot{q}^{\rho}}=0 \\
\frac{\partial H_{\sigma v}^{2}}{\partial t}=0 & \frac{\partial H_{\sigma v}^{2}}{\partial q^{\rho}}=0 & \frac{\partial H_{\sigma v}^{2}}{\partial \dot{q}^{\rho}}=0
\end{array}
$$

and

$$
\begin{aligned}
\varepsilon_{\theta \rho v} H_{\sigma \rho}^{0}+\varepsilon_{\theta \rho \sigma} H_{\rho v}^{0}+\varepsilon_{\theta \rho \kappa} \frac{\partial H_{\sigma v}^{0}}{\partial \ddot{q}^{\rho}} \ddot{q}^{\kappa}+\varepsilon_{\theta \rho \kappa} \frac{\partial H_{\sigma v}^{0}}{\partial \dddot{q}^{\rho}} \dddot{q}^{\kappa}=0 \\
\varepsilon_{\theta \rho \sigma} H_{\rho v}^{1}+\varepsilon_{\theta \rho \kappa} \frac{\partial H_{\sigma v}^{1}}{\partial \ddot{q}^{\rho}} \ddot{q}^{\kappa}+\varepsilon_{\theta \rho \kappa} \frac{\partial H_{\sigma v}^{1}}{\partial \dddot{q}^{\rho}} \dddot{q}^{\kappa}=0 \\
\varepsilon_{\theta \rho \sigma} H_{\rho v}^{2}+\varepsilon_{\theta \rho \kappa} \frac{\partial H_{\sigma v}^{2}}{\partial \ddot{q}^{\rho}} \ddot{q}^{\kappa}+\varepsilon_{\theta \rho \kappa} \frac{\partial H_{\sigma v}^{2}}{\partial \dddot{q}^{\rho}} \dddot{q}^{\kappa}=0
\end{aligned}
$$

From equalities (3.7), we get that $H_{\sigma v}^{0}, H_{\sigma v}^{1}$ and $H_{\sigma v}^{2}$ do not depend on $t, q^{\rho}, \dot{q}^{\rho}$. From (3.8), we get formula (3.6) and the vanishing of the components $H_{\sigma \nu}^{1}, H_{\sigma \nu}^{2}$.

Theorem 2. Let $H$ be a Helmholtz-like form (2.4) which is locally Helmholtz. H is invariant under the Galilei group if and only if the component $H_{\sigma \nu}^{0}$ of $H$ takes the form

$$
H_{\sigma v}^{0}=\varepsilon_{\sigma v \rho} c \ddot{q}^{\rho}
$$


where $c$ is constant and the components $H_{\sigma v}^{1}, H_{\sigma v}^{2}$ of $H$ vanish. The corresponding dynamical form takes the form

$$
E=-\frac{2}{3} \varepsilon_{\sigma \nu \rho} c q^{v} \ddot{q}^{\rho} \omega^{\sigma} \wedge \mathrm{d} t
$$

Proof. $H$ is locally Helmholtz, i. e., it corresponds to a system of differential equations, if and only if its components satisfy the following identities (see [7]):

$$
\begin{gathered}
\frac{\partial H_{\sigma v}^{2}}{\partial \dddot{q}^{\rho}}=0 \quad \frac{\partial H_{\sigma v}^{0}}{\partial \dddot{q}^{\rho}}+\frac{1}{2} \frac{\partial H_{\sigma \rho}^{2}}{\partial \dot{q}^{v}}=0 \\
\left(\frac{\partial H_{\sigma v}^{1}}{\partial \dddot{q}^{\rho}}-\frac{\partial H_{\sigma v}^{2}}{\partial \ddot{q}^{\rho}}\right)_{[v \rho]}=0 \quad\left(\frac{\partial H_{\sigma v}^{1}}{\partial \ddot{q}^{\rho}}-\frac{\mathrm{d}}{\mathrm{d} t} \frac{\partial H_{\sigma v}^{1}}{\partial \dddot{q}^{\rho}}\right)_{(v \rho)}=0 \\
\left(\frac{\partial H_{\sigma v}^{0}}{\partial \ddot{q}^{\rho}}+\frac{1}{2} \frac{\partial H_{\sigma v}^{2}}{\partial q^{\rho}}+\frac{1}{2} \frac{\partial H_{v \rho}^{2}}{\partial q^{\sigma}}-\frac{1}{2} \frac{\mathrm{d}}{\mathrm{d} t}\left(3 \frac{\partial H_{\sigma v}^{0}}{\partial \dddot{q}^{\rho}}+\frac{\partial H_{\sigma v}^{2}}{\partial \dot{q}^{\rho}}\right)\right)_{[v \rho]}=0 \\
\left(\frac{\partial H_{\sigma v}^{1}}{\partial \dot{q}^{\rho}}-\frac{1}{2} \frac{\mathrm{d}}{\mathrm{d} t} \frac{\partial H_{\sigma v}^{1}}{\partial \ddot{q}^{\rho}}+\frac{1}{2} \frac{\mathrm{d}^{2}}{\mathrm{~d} t^{2}} \frac{\partial H_{\sigma v}^{1}}{\partial \dddot{q}^{\rho}}\right)_{[v \rho]}=0 \\
\left(\frac{\partial H_{\sigma v}^{0}}{\partial \dot{q}^{\rho}}-\frac{1}{2}\left(\frac{\partial H_{\sigma \rho}^{1}}{\partial q^{v}}-\frac{\partial H_{v \rho}^{1}}{\partial q^{\sigma}}\right)-\frac{\mathrm{d}}{\mathrm{d} t}\left(\frac{\partial H_{\sigma v}^{0}}{\partial \ddot{q}^{\rho}}-\frac{1}{2} \frac{\partial H_{\sigma v}^{2}}{\partial q^{\rho}}\right)+\right. \\
\left.+\frac{\mathrm{d}^{2}}{\mathrm{~d} t^{2}} \frac{\partial H_{\sigma v}^{0}}{\partial \dddot{q}^{\rho}}\right)_{(v \rho)}=0 \\
\left(\frac{\partial H_{\sigma v}^{0}}{\partial q^{\rho}}-\frac{1}{3} \frac{\mathrm{d}}{\mathrm{d} t} \frac{\partial H_{\sigma v}^{0}}{\partial \dot{q}^{\rho}}+\frac{1}{3} \frac{\mathrm{d}^{2}}{\mathrm{~d} t^{2}}\left(\frac{\partial H_{\sigma v}^{0}}{\partial \ddot{q}^{\rho}}+\frac{\partial H_{\sigma v}^{2}}{\partial q^{\rho}}\right)-\right. \\
\left.-\frac{1}{3} \frac{\mathrm{d}^{3}}{\mathrm{~d} t^{3}} \frac{\partial H_{\sigma v}^{0}}{\partial \dddot{q}^{\rho}}\right)_{[v \rho]}=0 .
\end{gathered}
$$

The corresponding dynamical form is then in coordinates given by the formula (see [6]):

$$
E=\left(\tilde{E}_{\sigma}-\frac{\mathrm{d} \tilde{E}_{\sigma}^{1}}{\mathrm{~d} t}+\frac{\mathrm{d}^{2} \tilde{E}_{\sigma}^{2}}{\mathrm{~d} t^{2}}\right) \omega^{\sigma} \wedge \mathrm{d} t
$$

where

$$
\begin{aligned}
\tilde{E}_{\sigma} & =2 q^{v} \int_{0}^{1} H_{v \sigma}^{0}\left(t, u q^{\rho}, u \dot{q}^{\rho}, u \ddot{q}^{\rho}\right) u \mathrm{~d} u \\
& -\dot{q}^{v} \int_{0}^{1} H_{\sigma v}^{1}\left(t, u q^{\rho}, u \dot{q}^{\rho}, u \ddot{q}^{\rho}\right) u \mathrm{~d} u \\
& -\ddot{q}^{v} \int_{0}^{1} H_{\sigma v}^{2}\left(t, u q^{\rho}, u \dot{q}^{\rho}, u \ddot{q}^{\rho}\right) u \mathrm{~d} u
\end{aligned}
$$


and

$$
\begin{aligned}
& \tilde{E}_{\sigma}^{1}=q^{v} \int_{0}^{1} H_{v \sigma}^{1}\left(t, u q^{\rho}, u \dot{q}^{\rho}, u \ddot{q}^{\rho}\right) u \mathrm{~d} u, \\
& \tilde{E}_{\sigma}^{2}=q^{v} \int_{0}^{1} H_{v \sigma}^{2}\left(t, u q^{\rho}, u \dot{q}^{\rho}, u \ddot{q}^{\rho}\right) u \mathrm{~d} u .
\end{aligned}
$$

From equalities (3.7) and (3.11), we get that $H_{\sigma \nu}^{0}, H_{\sigma \nu}^{1}$ and $H_{\sigma \nu}^{2}$ do not depend on $t, q^{\rho}, \dot{q}^{\rho}, \dddot{q}^{\rho}$. From (3.8), we get (3.9). Substituting (3.9) into the (3.12), we get (3.10).

\section{ACKNOWLEDGEMENT}

Research supported by grants GA201/09/0981 of the Czech Science Foundation, SGS07/PrF/2012 of the University of Ostrava, RRC/04/2012 of the Moravian-Silesian region, and CZ-8/2009, TÉT 10-1-2011-0062 of Czech-Hungarian bilateral research cooperation.

\section{REFERENCES}

[1] M. Krbek and J. Musilová, "Representation of the variational sequence," Rep. Math. Phys., vol. 51, pp. 251-258, 2003.

[2] D. Krupka, "Variational sequences on finite order jet spaces," in World Scientific, Singapore, 1990, pp. 236-254.

[3] D. Krupka, "Variational sequences in mechanics," Calc. Var., vol. 5, pp. 557-583, 1997.

[4] D. Krupka and J. Šeděnková, "Variational sequences and Lepagean forms," in Differential Geometry and its Applications, Proc. Conf., Prague, 2004, J. Bureš, O. Kowalski, D. Krupka, and J. Slovák, Eds., Charles Univ., Prague, Czech Republic, 2005, pp. 605-615.

[5] O. Krupková, The Geometry of Ordinary Variational Equations, ser. Lecture Notes in Mathematics 1678. Berlin: Springer, 1997.

[6] O. Krupková and R. Malíková, "Helmholtz conditions and their generalization," Balkan Journal of Geometry and Its Applications (BJGA), vol. 15, no. 1, pp. 80-89, 2010.

[7] R. Malíková, "On a generalization of Helmholtz conditions," Acta Math. Univ. Ostraviensis, vol. 17, pp. 11-21, 2009.

Author's address

\section{Radka Malíková}

University of Ostrava, Department of Mathematics, 30. dubna 22, 70103 Ostrava, Czech Republic

E-mail address: radka.malikova@osu.cz 